\title{
Neuromuscular and postural control in visually and nonvisually impaired judo athletes: case study
}

\author{
Rafael Lima Kons ${ }^{1, *}$, Raphael Luiz Sakugawa', Mateus Rossato², Fernando Diefenthaeler', Daniele Detanico' \\ ${ }^{1}$ Biomechanics Laboratory, Federal University of Santa Catarina, Florianópolis, Brazil \\ ${ }^{2}$ Human Performance Laboratory, Federal University of Amazonas, Manaus, Brazil
}

This study aimed to analyze neuromuscular and postural control in visually and nonvisually impaired judo athletes. Two judo athletes, one visually impaired and the other nonvisually impaired, participated in the study. The athletes presented similar demographic, anthropometric, and judo-technical characteristics. They performed maximal isometric handgrip strength (dominant and nondominant hand), vertical jumps (countermovement jump [CMJ] and squat jump [SJ]), and center of pressure assessment in three positions: neutral, anteroposterior, and judo combat base (Migi-shizental). The main findings showed that the visually impaired athlete presented higher standing balance in the neutral and anteroposterior positions than non-visually impaired athlete (effect size [ES]>2.0). In the Migi-shizentai position, the disparity be- tween both athletes was reduced, particularly in the displacement area $(E S=0.52)$. The visually impaired athlete showed higher performance in the SJ but lesser performance in CMJ and handgrip strength tests than nonimpaired athlete $(E S>2.0)$. We concluded that the postural stability was higher in the visually impaired athlete in the neutral and anteroposterior position, but similar to the nonvisually impaired athlete in Migi-shizentaiposition, possible due to the influence of judo practice. Moreover, the visually impaired athlete showed higher performance in the SJ than nonvisually impaired.

Keywords: Combat sports, Balance, Center of pressure, Muscle strength, Vertical jumps

\section{INTRODUCTION}

Judo is an Olympic and Paralympic combat sport divided by sex and weight categories. The Paralympic judo is composed by visually impaired athletes who follow the same rules as Olympic judo with one main exception: the match is initiated with the athletes positioning their grips on the opponent's judogi, and this procedure is repeated every time combat is interrupted (Gutiérrez-Santiago et al., 2011). Judo matches are a constantly unstable environment, in which the athletes seek advantages by mainly disrupting the balance of their opponent to attack or contra-attack. According to Yoshitomi et al. (2006), judo athletes try to unbalance their opponent while maintaining their own stability to perform the throwing techniques. Thus, the balance is essential to efficiently throw the opponent with the back on the ground to obtain the high scores.
The center of pressure (COP) is an accurate measurement to estimate the postural stability (balance) in standing position, and represents the point where the resultant force acts on the support surface (Ruhe et al., 2010). Few studies have investigated the COP in judo athletes, as Santos et al. (2018) who verified increase in COP displacement and velocity over a simulated judo match, and Yoshitomi et al. (2006), who found better postural control system (lower COP displacement and velocity) in judo athletes compared to nonathletes after an unexpected external perturbation.

The standing balance may be affected by individual strength level. Dias et al. (2011) reported that $80 \%$ of COP variability can be associated to handgrip strength level in judo athletes, indicating that it generates changes in the postural stability. However, this study was performed with judo athletes who are not visually impaired. In general, the muscle strength and power in upper and lower limbs have been considered determining factors to judo
${ }^{\star}$ Corresponding author: Rafael Lima Kons (D) https://orcid.org/0000-0003-1615-5464 Federal University of Santa Catarina, Center of Sports, Biomechanics Laboratory, Florianópolis, Santa Catarina 88040-900, Brazil E-mail: rafakons0310@gmail.com

Received: November 9, 2018 / Accepted: January 21, 2019
This is an Open Access article distributed under the terms of the Creative Commons Attribution Non-Commercial License (http://creativecommons.org/licenses/by-nc/4.0/) which permits unrestricted non-commercial use, distribution, and reproduction in any medium, provided the original work is properly cited. 
performance (Detanico et al., 2012; Franchini et al., 2011; Franchini et al., 2013). For example, the isometric handgrip strength is widely required during judo matches to maintain the grip in the judogi and control the distance between the opponent (BonitchGóngora et al., 2012; Franchini et al., 2011; Kons et al., 2018). The muscle power of the lower limbs is relevant to perform several throwing techniques (Detanico et al., 2012; Torres-Luque et al., 2015) being correlated with the performance in judo-specific tests (Detanico et al., 2012).

In visually impaired judo athletes, it is possible to suggest that the matches present longer isometric handgrip strength actions than conventional judo matches, due to the higher grip time on the judogi (Gutiérrez-Santiago et al., 2011); however, no studies have analyzed the handgrip strength performance in both visually and non-visually impaired judo athletes. On the other hand, Loturco et al. (2017a) have verified higher values of muscle power in the lower limbs (i.e., vertical jump performance) in Olympic judo athletes compared to Paralympic ones, but no difference in maximal isometric strength exercises was found between them. According to the authors, the visual impairment may affect the performance in specific muscle power exercises due to the differences in visual-proprioceptive integration, which possibly leads to disparities between skill strategies during the exercises, but it is not yet clear.

Neuromuscular and postural control (standing balance) in judo athletes who are visually or nonvisually impaired has not been investigated yet. It is known that there is extreme difficulty in obtaining a large sample size particularly of visually impaired judo athletes, due to smaller number of visually impaired judo athletes. An alternative is to propose case studies, which has already been used in Paralympic judo (Loturco et al., 2017b) and other Paralympic sports (Antunes et al., 2017; Salvador et al., 2016). Thus, the aim of this study was to analyze the neuromuscular and postural control in visually and nonvisually impaired judo athletes.

\section{MATERIALS AND METHODS}

\section{Participants}

Two judo athletes, with one being visually impaired (congenital blindness) and the other not, participated in the study. The demographic, anthropometric, and judo-technical characteristics of the athletes are described in Table 1. The athletes evaluated were training regularly (physical, technical, and tactical training) for 4-5 times a week, and competing at the national level during the test period. Participants did not report musculoskeletal disorders or
Table 1. Demographic, anthropometric, and technical characteristics of the participants

\begin{tabular}{lccc}
\hline Variable & Visually impaired & Nonvisually impaired & $\Delta$ \\
\hline Age $(\mathrm{yr})$ & 21 & 20 & 1 \\
Height $(\mathrm{cm})$ & 185 & 183 & 2 \\
Body mass $(\mathrm{kg})$ & 92.3 & 91.5 & 0.8 \\
Body fat $(\%)$ & 20.9 & 21.7 & 0.8 \\
Judo level & Purple belt & Brown belt & - \\
Dominant foot side & Right & Right & - \\
Time of judo practice (yr) & 9 & 9 & 0 \\
Time of judo competitor (yr) & 7 & 8 & 1 \\
Level of competition & National & National & - \\
Weight category & $-90 \mathrm{~kg}$ & $-90 \mathrm{~kg}$ & - \\
Sport classification & $\mathrm{B} 1$ & $\mathrm{NC}$ & - \\
\hline
\end{tabular}

B1, visual acuity lower than LogMAR 2.6; NC, not applicable.

injuries that would influence their maximal performance, besides having trained judo systematically for the last 5 years and not being in a rapid weight loss period. Both participants were older than 18 years, and received a detailed verbal explanation of the purpose, methods, and potential risks/benefits of the study. Afterward, athletes signed a written informed consent form agreeing to participate in the investigation. The current study was approved by the Research Ethics Committee of the local university (CAAE: 63053516.4.0000.0121), in accordance with the Declaration of Helsinki.

\section{Handgrip strength protocol}

We adopted the procedures of American Society of Hand Therapy to testing the maximal isometric handgrip strength. Participants were seated and the shoulders were positioned in adduction and neutral rotations, and the elbow flexed at $90^{\circ}$ with the forearm in half-pronation and with a neutral grip. Athletes were instructed to perform maximum effort in a handgrip dynamometer (Carci, SH 5001 model) during 3 sec in the dominant hand with 30-sec rest period between each trial (three trials were performed). We used the highest value of handgrip strength as performance variable. The reliability of the handgrip assessment was tested and presented an intraclass correlation coefficient (ICC) of 0.92 for the dominant hand.

\section{Vertical jump assessment}

The muscle power of the lower limbs was accessed through two vertical jump protocols: countermovement jump (CMJ) and squat jump (SJ). Both tests are reliable and valid as they are widely used to estimate the muscle power of the lower extremities (Markovic 
et al., 2004). Before the CMJ and SJ assessment, participants performed exercises during a familiarization/warm-up period which include $30 \mathrm{sec}$ of hopping on a trampoline, three series of 10 hops on the ground, and five submaximal CMJs. After a 3-min resting period, athletes performed three $\mathrm{CMJ}$ on a piezoelectric force platform (model 9290AD, Kistler, Quattro Jump, Winterthur, Switzerland), which measures vertical ground reaction force sampling at $500 \mathrm{~Hz}$. Athletes started the CMJ protocol from a static standing position. They were instructed to perform a countermovement (descent phase), followed by a rapid and vigorous extension of the lower limb joints (ascent phase) and jump as high as possible. They also were recommended to flex their knees at $90^{\circ}$ during the eccentric-concentric phases. We provide a verbal feedback to the participants to encourage them to maintain a knee angle of approximately $90^{\circ}$ and maximum performance during the jump. Ten minutes afterward the CMJ protocol, the SJ was performed, that consisted of the athlete to jump from a static position, with the knees flexed at about $90^{\circ}$ and the hands on the waist. The SJ was performed without any countermovement and using only the concentric phase of the jump. We used the mean value of jump height, power output, and maximal force obtained in the concentric phase of the jump (within three trials). The reliability was tested among the three trials of the vertical jumps (CMJ and SJ) and presented an ICC ranging from 0.97-0.99 for jump height, power output and maximal force.

\section{Center of pressure}

The participants were assessed in three positions: neutral position, anteroposterior position (right foot forward according to the dominant side), and judo combat base (Migi-shizentai - right foot forward according to the dominant side with the grip on the judogi) (Fig. 1) using a force platform (OR6-6, AMTI, Watertown, MA, USA). The platform was placed on a stable surface on the floor to avoid signal distortion and noise. A reference point was placed $2 \mathrm{~m}$ in front of the athlete at eye level (for nonvisually impaired athlete). Both participants were informed about the importance of maintaining the positions fixed and were asked to avoid any body movement. Three consecutive 60 -sec repetitions were performed (Carpenter et al., 2001) at three different protocols in a random order. Displacement signals were smoothly digitally by a recursive Butterworth low pass filter with a $10-\mathrm{Hz}$ cutoff frequency to assure that $99 \%$ of power spectral density was below this threshold (Clark et al., 2010). Raw data were recorded at a frequency of $40 \mathrm{~Hz}$. We removed the first $10 \mathrm{sec}$ of each trial of the analysis to avoid any interference from delayed stabilization of re-

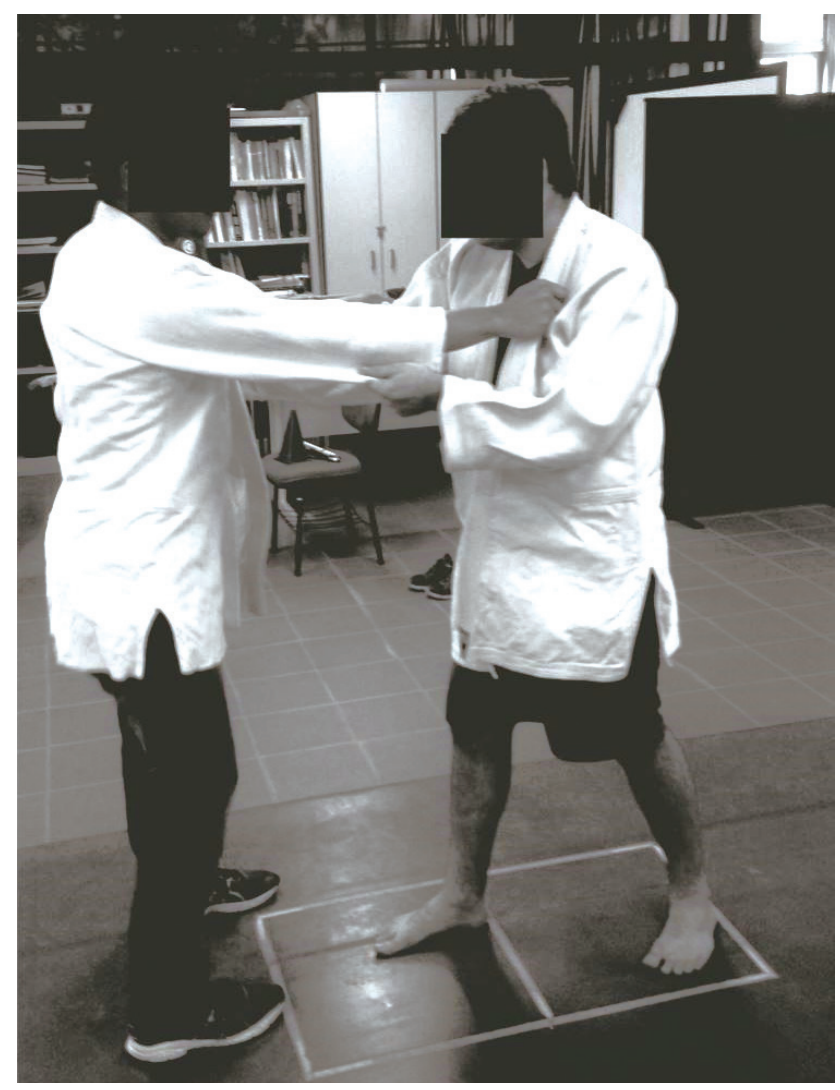

Fig. 1. Athlete's position during the center of pressure assessment in the Migi-shizentai position.

cording equipment after the person stepped onto the force platform (Raymakers et al., 2005).

According to Ruhe et al. (2010) and Santos et al. (2018) recommendations, three types of COP parameters were assessed: positioning, velocity, and dispersion. COP positioning was determined by computing the composite COP displacement length, area, and velocity. Additionally, $\mathrm{COP}$ positioning in the anteroposterior (Xaxis) and mediolateral axis (Y-axis), mean position ( $\mathrm{X}_{\text {MEAN }}$ and $\left.\mathrm{Y}_{\mathrm{MEAN}}\right)$, velocity ( $\mathrm{X}_{\mathrm{VEL}}$ and $\mathrm{Y}_{\mathrm{VEL}}$ ), and resultant $\left(\mathrm{Y}_{\mathrm{RES}}\right)$ for length and velocity, were also computed and reported. Data were collected at a sampling frequency of 2,000 $\mathrm{Hz}$ and were smoothed using a fifth-order, zero phase, low pass Butterworth digital recursive filter with a frequency range set of $10 \mathrm{~Hz}$. Area of COP was estimated by fitting an ellipse that encompasses $95 \%$ of COP data. Off-line data analyses were developed with custom-written scripts (R 2014a, MathWorks, Natick, MA, USA).

\section{Statistical analysis}

Data are reported as mean \pm standard deviation. The differences between the means $(\Delta)$ and effect size (ES) were calculated in order 
to analyze the biomechanical variables between the athletes. The GPower3.1 software (University of Kiel, Kiel, Germany) was used to calculate ES, and the criterion proposed by Hopkins (2002) was used to classify ES: $0.0-0.2$, trivial; 0.21-0.6, small; 0.61-1.2, moderate; $1.21-2.0$, large; and 2.1-4.0, very large.

\section{RESULTS}

In the neutral and anteroposterior position, all results indicated lesser COP parameters (length, velocity, and area [very large effect]) for the visually impaired athlete. The disparity between the athletes seems to be reduced when assuming the judo position

Table 2. Center of pressure in in three different positions (neutral, anteroposterior, and Migi-shizental) in visually and nonvisually impaired athletes

\begin{tabular}{|c|c|c|c|c|c|}
\hline Variable & $\begin{array}{c}\text { Nonvisually } \\
\text { impaired }\end{array}$ & $\begin{array}{l}\text { Visually } \\
\text { impaired }\end{array}$ & $\Delta$ & ES & Classification \\
\hline \multicolumn{6}{|c|}{ Neutral position } \\
\hline \multicolumn{6}{|c|}{ Length (cm) } \\
\hline$X_{\text {MEAN }}$ & $29.5 \pm 2.20$ & $20.5 \pm 1.42$ & 9.0 & 4.86 & Very large \\
\hline$Y_{\text {MEAN }}$ & $36.0 \pm 2.34$ & $29.0 \pm 1.02$ & 7.0 & 3.87 & Very large \\
\hline$X, Y_{\text {RES }}$ & $51.1 \pm 3.13$ & $39.1 \pm 1.92$ & 12.0 & 4.62 & Very large \\
\hline \multicolumn{6}{|c|}{ Velocity (cm/sec) } \\
\hline$X_{\text {VEL }}$ & $0.98 \pm 0.07$ & $0.68 \pm 0.04$ & 0.30 & 5.26 & Very large \\
\hline$Y_{V E L}$ & $1.20 \pm 0.07$ & $0.96 \pm 0.03$ & 0.24 & 4.45 & Very large \\
\hline$X, Y_{\text {RES }}$ & $1.70 \pm 0.10$ & $1.30 \pm 0.06$ & 0.40 & 4.85 & Very large \\
\hline Area $(\mathrm{cm})$ & $3.46 \pm 1.36$ & $0.87 \pm 0.30$ & 2.59 & 2.63 & Very large \\
\hline \multicolumn{6}{|c|}{ Anteroposterior position } \\
\hline \multicolumn{6}{|c|}{ Length $(\mathrm{cm})$} \\
\hline$X_{\text {MEAN }}$ & $48.7 \pm 2.80$ & $17.7 \pm 0.28$ & 31.0 & 15.5 & Very large \\
\hline$Y_{\text {MEAN }}$ & $46.7 \pm 2.19$ & $22.1 \pm 0.32$ & 24.6 & 15.7 & Very large \\
\hline$X, Y_{\text {RES }}$ & $65.8 \pm 4.60$ & $31.0 \pm 0.51$ & 34.8 & 10.6 & Very large \\
\hline \multicolumn{6}{|c|}{ Velocity (cm/sec) } \\
\hline XVEL & $1.21 \pm 0.14$ & $0.59 \pm 0.09$ & 0.62 & 5.26 & Very large \\
\hline Yvel & $1.55 \pm 0.07$ & $0.73 \pm 0.01$ & 0.82 & 16.4 & Very large \\
\hline$X, Y_{\text {RES }}$ & $2.19 \pm 0.15$ & $1.03 \pm 0.01$ & 1.16 & 10.9 & Very large \\
\hline Area $(\mathrm{cm})$ & $4.09 \pm 0.98$ & $0.58 \pm 0.14$ & 3.51 & 5.01 & Very large \\
\hline \multicolumn{6}{|c|}{ Migi-shizentai } \\
\hline \multicolumn{6}{|c|}{ Length $(\mathrm{cm})$} \\
\hline$X_{\text {MEAN }}$ & $48.7 \pm 2.80$ & $53.0 \pm 10.9$ & -4.30 & 0.54 & Small \\
\hline$Y_{\text {MEAN }}$ & $77.8 \pm 5.01$ & $58.8 \pm 14.7$ & 19.0 & 1.73 & Large \\
\hline$X, Y_{\text {RES }}$ & $98.1 \pm 3.91$ & $87.2 \pm 19.1$ & 10.9 & 0.79 & Moderate \\
\hline \multicolumn{6}{|c|}{ Velocity (cm/sec) } \\
\hline XVEL & $1.62 \pm 0.09$ & $1.76 \pm 0.36$ & -0.14 & 0.53 & Small \\
\hline $\mathrm{Y}_{\mathrm{VEL}}$ & $2.59 \pm 0.16$ & $1.96 \pm 0.49$ & 0.63 & 1.72 & Large \\
\hline$X, Y_{\text {ReS }}$ & $3.27 \pm 0.13$ & $2.90 \pm 0.63$ & 0.37 & 0.81 & Moderate \\
\hline Area $(\mathrm{cm})$ & $2.29 \pm 0.48$ & $2.98 \pm 1.80$ & -0.69 & 0.52 & Small \\
\hline
\end{tabular}

ES, effect size; X, Y, Cartesian coordinate; VEL, velocity; RES, resultant.
Migi-shizentai, mainly for $\mathrm{X}_{\mathrm{MEAN}}, \mathrm{X}_{\mathrm{VEL}}$, and area, showing similar balance in these variables. Despite this, the visually impaired athlete presented higher balance for length $\left(\mathrm{Y}_{\mathrm{MEAN}} ; \mathrm{X}, \mathrm{Y}_{\mathrm{RES}}\right)$ and velocity $\left(\mathrm{Y}_{\mathrm{VEL}} ; \mathrm{X}, \mathrm{Y}_{\mathrm{RES}}\right)$ (Table 2).

Fig. 2 shows the COP behavior in the neutral position, anteroposterior, and Migi-sbizentai in visually and nonvisually impaired athletes, ratifying the findings that the visually impaired athlete showed lesser displacement and consequently better standing bal-
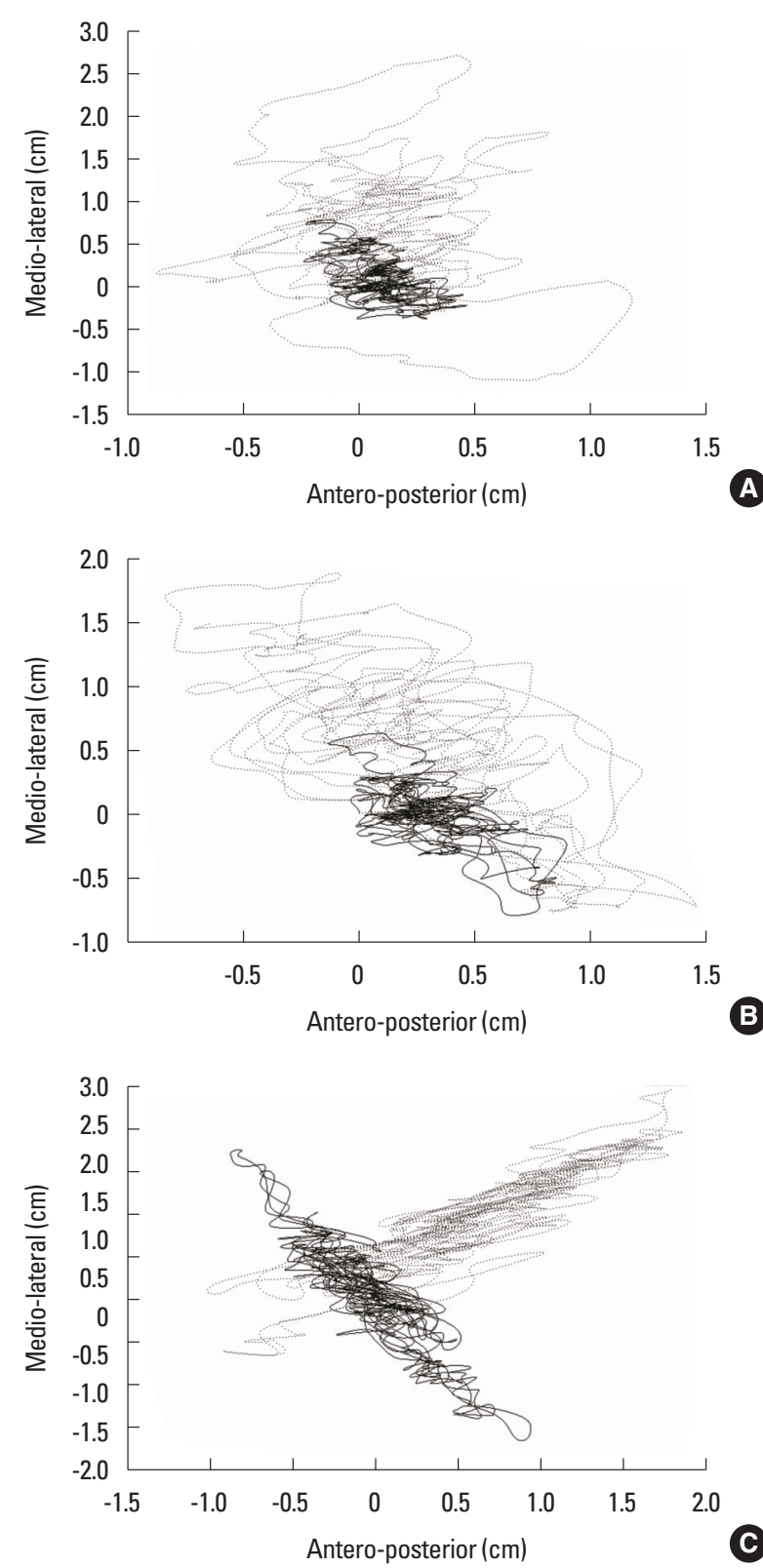

Fig. 2. Center of pressure behavior in the neutral position (A), anteroposterior $(\mathrm{B})$, and Migi-shizentai (C) in visually (full line) and nonvisually impaired athletes (dotted line). 


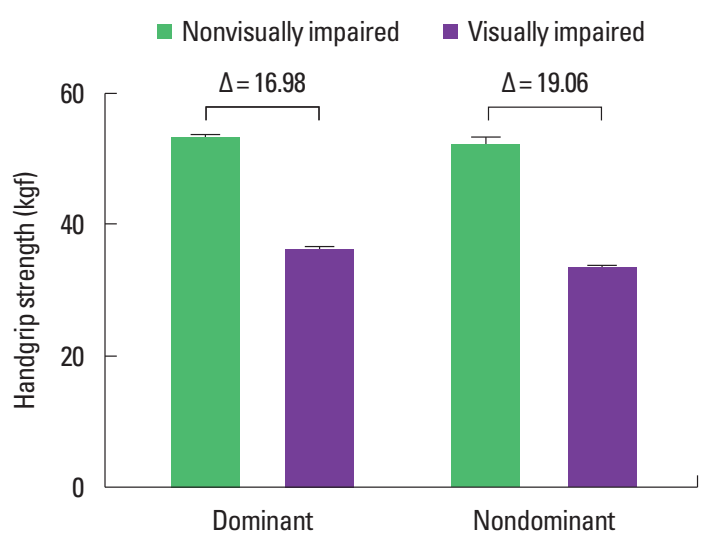

Fig. 3. Maximal isometric handgrip strength in visually and nonvisually impaired athletes in dominant and nondominant hand.

ance than non-impaired athlete in the first two positions, reducing the difference in the judo-specific position.

Fig. 3 presents the maximal handgrip strength in visually and nonvisually impaired athletes at dominant and nondominant hand. The results indicate higher values for the nonvisually impaired athlete in both dominant ( $\mathrm{ES}=24.01$, very large) and nondominant hand ( $\mathrm{ES}=27.03$, very large).

The analysis of $\mathrm{CMJ}$ indicated very large effect for jump height $(\mathrm{ES}=2.18)$ and power output $(\mathrm{ES}=2.91)$ in favor of the visually impaired athlete (Fig. 4). On the other hand, in the SJ, the ES analysis showed very large effect for power output $(\mathrm{ES}=5.14)$ and maximal force ( $E S=2.02)$ in favor of the nonvisually impaired (Fig. 4).

\section{DISCUSSION}

The purpose of the present study was to analyze the neuromuscular and postural control in visually and nonvisually impaired judo athletes. Both athletes show similar demographic, anthropometric, and judo-technical characteristics. In our study, we found that the visually impaired athlete showed higher balance (lesser length, velocity, and area) in the neutral and anteroposterior positions than the nonvisually impaired athlete. In the Migi-shizentai position, the disparity between both athletes was reduced, particularly in the area of displacement. Furthermore, the visually impaired athlete has shown higher performance in the SJ (power output and maximal force) but lesser performance in the CMJ (jump height and power output) and handgrip strength test compared to the nonimpaired athlete.

The postural control during judo matches is an important aspect in the execution of throwing techniques (Santos et al., 2018;
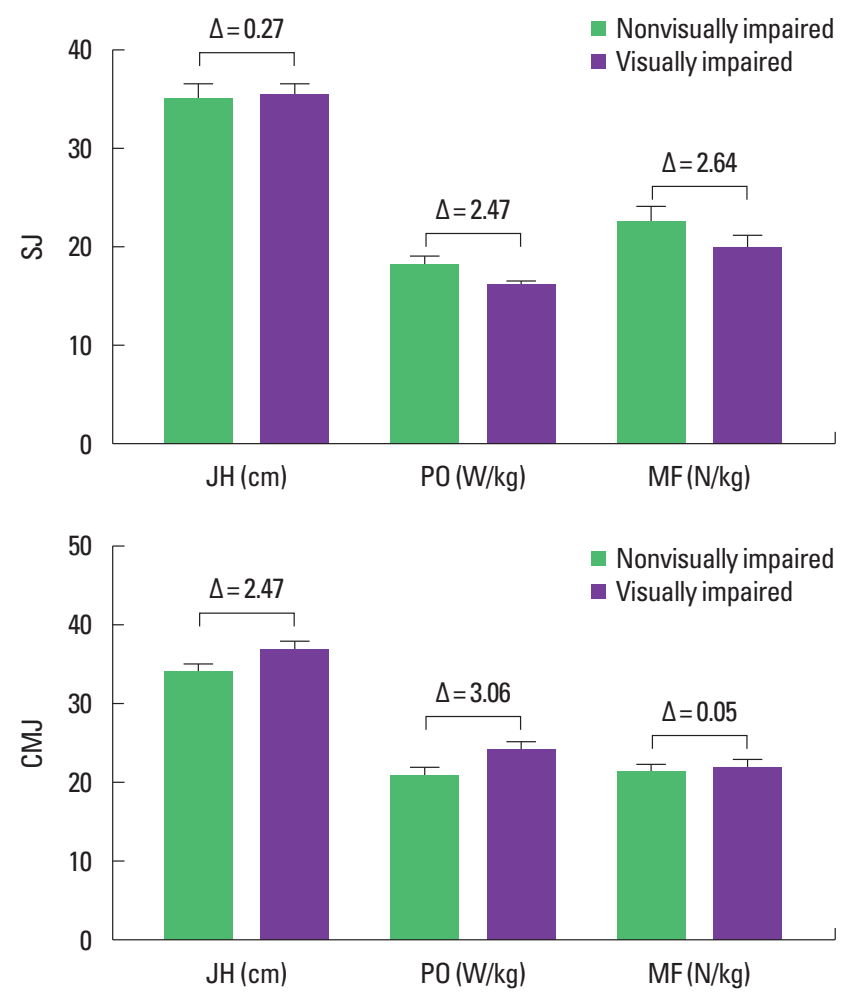

Fig. 4. Jump height (JH), power output (PO), and maximal force (MF) obtained from squat jump (SJ) and countermovement jump (CMJ) in visually and nonvisually impaired athletes.

Yoshitomi et al., 2006) or during the defense in both judo modalities (conventional and with visual impairment). However, in visually impaired athletes, the postural control has a greater peculiarity due to the low or no vision of the athletes (Krabben et al., 2018). In this case, other postural control systems (vestibular and somatosensory) seem to assume a more prominent role in maintaining balance, and thus compensate for the weak or absent visual input (Schwesig et al., 2011). This fact may explain the higher balance in the neutral and anteroposterior position in the visually impaired. Moreover, the lesser variation of the COP area between the two athletes in the judo-specific position (Migui-shinzetai) may show an adaptation to the actions involving judo, e.g., during the kuzushi (imbalance) that is essential in the execution of judo throwing techniques.

The vertical jump performance (CMJ and SJ) is the best parameter to assess the muscle power of the lower limbs (Markovic et al., 2004) and has been used frequently in judokas non-visually impaired (Franchini et al., 2011) and visually impaired (Loturco et al., 2017a; Loturco et al., 2017b). In our study, the visually impaired athlete has shown higher effect for power output and maximal force in the SJ compared to the non-visually impaired, while 
in the CMJ, the opposite was found (jump height and power output were higher in the visually impaired). Loturco et al. (2017a) evaluated judo athletes from Brazilian Olympic and Paralympic teams and verified higher values in jump height and maximum propulsive power in the SJ, bench press, and standing barbell row in the Olympic team than Paralympic; but the maximal isometric strength in the half-squat and bench press did not differ between the groups. It is possible to suggest that the visual impairment may affect the performance in specific muscle power exercises, as there is no field of view, the perception of safety and balance decreases (Makris et al., 1993). Thus, the motion pattern during the jump execution seems safer in the SJ than CMJ, which could explain the higher maximal force and power output produced during the propulsive phase of the $S J$ by the visually impaired. In addition, the differences in the visual-proprioceptive aspects between the athletes possibly lead to disparities between their performance strategies, mainly because the visual stimulus assists in tasks involving higher velocity (e.g., tasks involving the shortening-stretch cycle, as the $\mathrm{CMJ}$ ).

Maximal isometric handgrip strength is one of the determining factors of judo performance (Calmet et al., 2010; Franchini et al., 2011), especially during grip disputes and moments that precede the throwing techniques (Kons et al., 2018). The findings of this study demonstrate a very large effect for both hands in the nonvisually impaired athlete compared to the visually impaired. It is worth noting that the handgrip strength test is a generic assessment and does not approach specific actions of the sport, although it is widely used to evaluate judo athletes, as it measures the forearm strength that represents the moments of grip on the judogi (Bonitch-Góngora et al., 2012; Franchini et al., 2011; Kons et al., 2018). The visually impaired athlete starts the match by positioning his/her grip on the opponent's judogi, and this procedure is repeated every time combat is interrupted (Gutiérrez-Santiago et al., 2011). Thus, a speculative explanation is that the nonvisually impaired athletes have other possibilities to perform the grip during matches, i.e., in different parts of the judogi jacket, thus allowing higher adaptations to the forearm and finger flexor muscles than the visually impaired athletes.

Finally, the case study was composed only by two athletes, which limited our statistical analyses. However, this is the first study that analyzed neuromuscular and postural control parameters in visually and nonvisually impaired athletes with similar demographic, anthropometric, and technical characteristics. Thus, we recommend to the coaches and sports scientists to monitor the balance, mainly in judo-specific postures, as well as to use muscle power of lower limbs assessments that increase the perception of safety in visually impaired athletes, e.g. SJ instead of CMJ.

We conclude that the visually impaired athlete presented higher postural stability (balance), mainly in the neutral and anteroposterior positions, than the nonvisually impaired athlete. In the Migi-shinzentai position, the postural stability was higher in the COP length and velocity in the visually impaired judoka, but not in the area, showing a possible influence of judo practice in the standing balance in the judo-specific position. Considering the neuromuscular aspects, the visually impaired athlete presented higher performance in the SJ but lesser performance in the CMJ and isometric handgrip strength test than the nonvisually impaired.

\section{CONFLICTS OF INTEREST}

No potential conflict of interest relevant to this article was reported.

\section{ACKNOWLEDGMENTS}

The authors would like to thank all volunteer for their participation in the study.

\section{REFERENCES}

Antunes D, Rossato M, Kons RL, Sakugawa RL, Fischer G. Neuromuscular features in sprinters with cerebral palsy: case studies based on paralympic classification. J Exerc Rehabil 2017;13:716-721.

Bonitch-Góngora JG, Bonitch-Domínguez JG, Padial P, Feriche B. The effect of lactate concentration on the handgrip strength during judo bouts. J Strength Cond Res 2012;26:1863-1871.

Calmet M, Miarka B, Franchini, E. Modeling of grasps in judo contests. Int J Perform Anal Sport 2010;10:229-240.

Carpenter MG, Frank JS, Winter DA, Peysar GW. Sampling duration effects on centre of pressure summary measures. Gait Posture 2001;13: 35-40.

Clark RA, Bryant AL, Pua Y, McCrory P, Bennell K, Hunt M. Validity and reliability of the Nintendo Wii Balance Board for assessment of standing balance. Gait Posture 2010;31:307-310.

Detanico D, Dal Pupo J, Franchini E, dos Santos SG. Relationship of aerobic and neuromuscular indexes with specific actions in judo. Sci Sport 2012;27:16-22.

Dias JA, Külkamp W, Wentz MD, Ovando AC, Borges NG Jr. Effect of handgrip on the balance of judokas. Motriz: Rev Educ Fis (Online) 
2011;17:244-251.

Franchini E, Artioli GG, Brito CJ. Judo combat: time motion analysis and physiology. Int J Perform Anal Sport 2013;13:624-641.

Franchini E, Del Vecchio FB, Matsushigue KA, Artioli GG. Physiological profiles of elite judo athletes. Sports Med 2011;41:147-166.

Gutiérrez-Santiago A, Prieto I, Camerino O, Anguera T. The temporal structure of judo bouts in visually impaired men and women. J Sports Sci 2011;29:1443-1451.

Hopkins WG. A scale of magnitudes for effect statistics. Sport Sci [Internet]. 2002 Jun [cited 2018 Sep 20]. Available from: https://www.sportsci.org/resource/stats/effectmag.html.

Kons RL, Pupo JD, Ache-Dias J, Garcia T, da Silva RR, Katicips LFG, Detanico D. Effect of official judo matches on handgrip strength and perceptual responses. J Exerc Rehabil 2018;14:93-99.

Krabben KJ, van der Kamp J, Mann, DL. Fight without sight: the contribution of vision to judo performance. Psychol Sport Exerc 2018;37:157163.

Loturco I, Nakamura FY, Winckler C, Bragança JR, da Fonseca RA, Moraes-Filho J, Zaccani WA, Kobal R, Cal Abad CC, Kitamura K, Pereira LA, Franchini E. Strength-power performance of visually impaired Paralympic and Olympic judo athletes from the Brazilian National Team: a comparative study. J Strength Cond Res 2017a;31:743-749.

Loturco I, Pereira LA, Winckler C, Bragança JR, da Fonseca RA, Kobal R, Cal Abad CC, Kitamura K, Nakamura FY, Franchini E. Performance changes of elite paralympic judo athletes during a Paralympic games cycle: a case study with the Brazilian National Team. J Hum Kinet 2017b;60:217-224.

Makris VI, Yee RD, Langefeld CD, Chappell AS, Slemenda CW. Visual loss and performance in blind athletes. Med Sci Sports Exerc 1993;25:
265-269.

Markovic G, Dizdar D, Jukic I, Cardinale M. Reliability and factorial validity of squat and countermovement jump tests. J Strength Cond Res 2004;18:551-555.

Raymakers JA, Samson MM, Verhaar HJ. The assessment of body sway and the choice of the stability parameter(s). Gait Posture 2005;21:48-58.

Ruhe A, Fejer R, Walker B. The test-retest reliability of centre of pressure measures in bipedal static task conditions--a systematic review of the literature. Gait Posture 2010;32:436-445.

Salvador AF, Schubert KR, Cruz RS, Corvino RB, Pereira KL, Caputo F, de Oliveira MF. Bilateral muscle strength symmetry and performance are improved following walk training with restricted blood flow in an elite paralympic sprint runner: Case study. Phys Ther Sport 2016;20:1-6.

Santos L, Fernández-Río J, Iglesias-Soler E, Blanco-Traba M, Jakobsen MD, González-Díez V, Franchini E, Gutiérrez C, Dopico-Calvo X, Carballeira-Fernández E, Amonette W, Suman O. Postural control and physiological responses to a simulated match in U-20 judo competitors. Sports Biomech 2018 May 21:1-14 [Epub]. https://doi.org/10.1080/ 14763141.2018.1461237.

Schwesig R, Goldich Y, Hahn A, Müller A, Kohen-Raz R, Kluttig A, Morad $Y$. Postural control in subjects with visual impairment. Eur J Ophthalmol 2011;21:303-309.

Torres-Luque G, Hernandez-Garcia R, Garatachea N, Nikolaidis PT. Anthropometric characteristics and neuromuscular function in young judo athletes by sex, age and weight category. Sport Sci Health 2015; 11:117-124.

Yoshitomi SK, Tanaka C, Duarte M, Lima F, Morya E, Hazime F. Postural responses to unexpected external perturbance in judoists of different ability levels. Braz J Sports Med 2006;12:159-163. 